\title{
Globalización económica, postmodernidad y sistema educativo: contradicciones y alternativas desde una Educación Física crítica
}

\author{
Economic globalization, postmodernism, and educational system: \\ Contradictions and alternatives from a critical physical education perspective
}

\author{
Bastian Carter-Thuillier ${ }^{a}$, Alberto Moreno Doña ${ }^{b}$

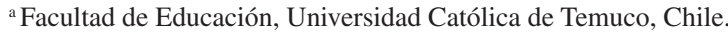 \\ Correo electrónico: bcarter@uct.cl \\ ${ }^{\mathrm{b}}$ Facultad de Medicina, Escuela de Educación Parvularia, Universidad de Valparaíso, Chile. \\ Correo electrónico: alberto.moreno@uv.cl
}

\begin{abstract}
La globalización económica se construye ideológicamente desde el neoliberalismo, atravesando toda la realidad social; esto incluye a un sistema educativo que enfrenta actualmente diferentes contradicciones en términos axiológicos, sociales, políticos y culturales. Dicha tensión encuentra una posible explicación en el anacronismo de dicho sistema, que promueve prácticas y valores modernos en plena era postmoderna. Este artículo tiene por objetivo analizar la relación existente entre sistema educativo y globalización económica en las condiciones particulares de la posmodernidad, intentando además comprender críticamente sus implicancias para el campo de la Educación Física; ello a partir del análisis y reflexión de antecedentes teóricos asociados a la temática. El manuscrito se organiza en tres apartados, primero a modo introductorio se presenta la problematización; en segundo término se analiza y describe la relación entre globalización económica, postmodernidad y educación; en el apartado final se presentan propuestas críticas desde la Educación Física y el deporte.
\end{abstract}

Palabras clave: pedagogía crítica, condición postmoderna, modelo neoliberal, prácticas tecnocráticas.

\begin{abstract}
Economic globalization is built ideologically from neoliberalism, crossing all social reality; this includes the education system that currently faces various contradictions in axiological, social, political, and cultural terms. This tension has a possible explanation in the anachronism of system, which promotes practical and modern values in the postmodern age. This article aims to analyze the relationship between education system and economic globalization in the particular conditions of postmodernism, trying to understand from a critical approach the implications for the physical education field based on analysis and reflection of theoretical background associated with the topic. The manuscript is organized into three sections. First, the problematization is presented; second the relationship between economic globalization, postmodernity and education is analyzed and described; in the final section critical proposals from physical education and sport are presented.
\end{abstract}

Key words: critical pedagogy, postmodern condition, neoliberal model, technocratic practices. 


\section{INTRODUCCIÓN}

La agudización de la crisis global en los ámbitos sociales, económicos, culturales y ambientales, es para Abril (2015) una crisis sistémica que comenzó como un problema financiero y acabó derivando en una crisis civilizatoria. Esta última ha detonado el cuestionamiento y desconfianza de los diversos agentes sociales respecto a los procesos globales y un déficit de credibilidad respecto al triunfalismo neoliberal, que se presentaba durante las últimas décadas como un flujo económico continuo sin alternativas necesarias por parte de sus defensores (Munck, 2010).

La globalización en su totalidad es un fenómeno histórico más complejo y poderoso de lo que habitualmente se exhibe tanto en los análisis mediático-sociales, como en el campo económico (Sloterdijk, 2014). La transnacionalización y la virtualización del conocimiento, así como de las instituciones encargadas de su producción, reproducción y transformación (como las educativas) (Rengifo-Millán, 2015) ha dado paso a un escenario de relativización de los saberes y valores históricamente vinculados al "campus" educativo.

Para Rivero (2013) el neoliberalismo, la globalización y la educación forman parte de una sola totalidad social, política, económica y cultural no exenta de contradicciones; paradojas que según el mismo autor promueven tanto la legitimación, como superación de dicho escenario. En dicho contexto, Teodoro (2014) manifiesta que durante las últimas décadas, los discursos y políticas públicas en el campo de la educación han dado pie a la reducción del concepto "democracia" en el sistema educativo, siendo reemplazado por prácticas de consumo económico; ocurriendo lo mismo con el de "ciudadanía" que ha sido paulatinamente sustituido por el individualismo. Esto ha potenciado y posibilitado la transferencia de éste sector hacia el mercado en medio de la globalización neoliberal. En dicho plano, Cardona (2014) expresa que existe una distancia insondable entre las políticas educativas, modelos pedagógicos, lineamientos curriculares y la estandarización del conocimiento que se ha generado desde el metarrelato transmoderno de la globalización, lo que también ha conllevado el desarrollo de prácticas docentes anacrónicas.

Afrontar los desafíos educativos actuales sin considerar los procesos sociales, políticos y económicos es difícil, más aún si ellos se enmarcan en un escenario global y hegemonizado por el proyecto neoliberal (Abril, 2015). La complejidad aumenta si consideramos además la dimensión histórica en la que esos fenómenos transcurren, entendiendo las tensiones entre un sistema educativo que promueve valores modernos, en pleno desarrollo de la era postmoderna (Hargreaves, 2005; Ovejero, 2004).

Este fenómeno y cada uno de sus componentes antes mencionados atraviesan de forma transversal a toda la realidad educativa y lógicamente también cada uno de sus campos, incluyendo entre ellos a la Educación Física (EF). En consideración de lo antes expuesto, el presente artículo tiene por objeto analizar la relación existente entre sistema educativo y globalización económica en las condiciones particulares de la postmodernidad, centrándose en las contradicciones intrínsecas de dicho escenario e intentando comprender críticamente sus implicancias y posibilidades para el campo específico de la EF. 


\section{GLOBALIZACIÓN ECONÓMICA, EDUCACIÓN Y POSTMODERNIDAD}

La globalización económica, se expresa como una continua tendencia a la extensión de los mercados, sobrepasando las barreras nacionales de origen para tornarse en un fenómeno mundial. Dicho fenómeno posee intrínsecamente inmerso el neoliberalismo como ideología, impregnando a todas las capas de la sociedad (Ovejero, 2004) y por lo tanto a todas las instituciones que forman parte de la estructura de la misma, donde el sistema educativo ocupa un rol preponderante (Apple, 2013a; Bourdieu \& Passeron, 1990; Bourdieu, 2010).

El fenómeno de la globalización económica se desarrolla en el marco histórico de la postmodernidad (Ovejero, 2008). La condición postmoderna emerge desde el campo científico a través de Lyotard $(2000)^{1}$, quien expresa una crítica respecto a los augurios modernos incumplidos durante el siglo XX y a la propia actividad científica, aduciendo la necesidad de avanzar hacia una práctica científica con una dimensión emancipadora, que debe enfrentar nuevos marcos de legitimación (Ruiz de Samaniego, 2004). Este cambio se vio reforzado por las aportaciones de Kuhn $(2010)^{2}$ en la década del setenta y su estructura de las revoluciones científicas que derriba la visión clásica e idealizada de la ciencia, a lo que se suma la epistemología anarquista de Feyerabend (1986, 1990), a través de su "Tratado contra el método" realizando cuestionamientos severos a la posición racionalista científica.

La postmodernidad no posee sólo una dimensión científica, sino que también tiene características políticas, económicas, técnicas y un orden hegemónico particular (Ovejero, 2004). Esta situación ha facilitado la generación de diversos cambios sociales, los cuales van desde la aparición de la "sociedad de consumo" y el fortalecimiento de la democracia formal en occidente, hasta el aumento en la brecha de inequidad económica entre los Estados más ricos y pobres, así como entre sus propios habitantes (Londoño, 2008; Ovejero, 2004). A esto se suma el aumento en los flujos migratorios como consecuencia de la globalización económica, la internacionalización de los medios de comunicación y los desequilibrios en el bienestar material (Pereda, Actis \& Prada, 2000). Todos fenómenos que en su totalidad acaban repercutiendo y manifestándose en el espacio social y escolar a través de la conformación de sociedades multiculturales (Caro, 2013; Flecha \& Puigvert, 2002).

El sistema educativo y sus procesos de enseñanza constituyen la instancia en la que se produce la transmisión y ejercitación de aquellos valores sociales que se consideran “positivos" para cada época (Díez, 2010). Ello entendiendo que éstos valores se reproducen y/o cambian en cada contexto socio-histórico, siendo comúnmente determinados según la cosmovisión cultural hegemónica y la ideología dominante (Freire, 2012) ${ }^{3}$. Esto último suele implicar en la mayoría de las oportunidades que dichos valores se acepten y practiquen sin ser objeto de cuestionamiento alguno, configurándose a partir de una lógica universalizada y absoluta (Prior, 2002). Esta función desempeñada por el sistema educativo requiere de una constante reflexión axiológica (en el significado original del propio término); con esto hacemos referencia a la necesidad de cuestionar la naturaleza de los valores y los juicios valorativos que se instauran y reproducen en la escuela.

Obra originalmente publicada en 1979, se referencia la edición empleada para redacción del presente artículo.

Obra originalmente publicada en 1962, se referencia la edición empleada para redacción del presente artículo.

Obra originalmente publicada en 1970, se referencia la edición empleada para redacción del presente artículo. 
El sistema educativo se encuentra hoy en una contradicción ontológica e histórica, puesto que a pesar de ser una institución de alta trascendencia social, se encuentra ligada a los valores de la modernidad; ello a pesar de encontrarse en la actualidad en pleno desarrollo la era postmoderna (Hargreaves, 2005; Martín, 2014; Ovejero, 2002), contexto que se contrapone en diferentes aspectos a su período antecesor, y que a su vez, convive con la ideología neoliberal y la lógica del mercado regulando en gran parte el acontecer social (Ovejero, 2004). En términos resumidos, la escuela es una institución moderna en la postmodernidad, por consecuencia directa "descontextualizada" (Ovejero, 2002) y en crisis con su entorno.

En relación a lo expuesto anteriormente, la escuela continúa exaltando y promoviendo los valores de la modernidad e inclusive algunos propios del periodo preindustrial (Zeraoui, 2000), tornándose con ello una institución social descontextualizada. Como consecuencia a ésta situación contradictoria, la escuela coarta las posibilidades de promoción social a individuos pertenecientes a grupos desfavorecidos que participan de ella, beneficiando a los pertenecientes a sectores privilegiados (Ovejero, 2004). Esto ha sido denunciado en años anteriores, enfatizándose cómo el sistema educativo contribuye a la reproducción de la estructura social existente e inclusive a la restricción del acceso al capital cultural a favor de algunos grupos determinados que forman parte de las elites, en desmedro de los demás, lo que evidentemente producirá desequilibrio en las relaciones de poder y la vida social de estos sujetos (Apple, 2013b; Bourdieu, 2007; Bourdieu \& Passeron, 1990, 20094; Foucault, 20075).

Las características reproductivas del sistema educativo, se ven aún más potenciadas en la actualidad, puesto que los valores que en él se practican y pregonan (valores modernos, tales como: el trabajo, el ahorro, el esfuerzo, etc.) están en conflicto constante con una sociedad asediada por la irrupción tecnológica y una publicidad que insta a practicar valores opuestos a los que se promueven a nivel educativo formal (Ovejero, 2002). A este escenario se suman además el consumismo, el darwinismo y la exclusión social, así como los distintos tipos de discriminación postmoderna (Flecha, 2001), que dan pie al distanciamiento y aislamiento entre las diversas manifestaciones de diversidad cultural.

En pocas palabras, actualmente tenemos una escuela moderna (en consecuencia anacrónica) que atraviesa una contradicción intrínseca, perdiendo inclusive protagonismo en los procesos de socialización de los niños, y que encima tiene como usuarios a estudiantes postmodernos (Ovejero, 2004; Flecha y Puigvert, 2002).

A pesar del complejo momento que atraviesa el sistema educativo, los centros escolares continúan siendo una instancia con un alto potencial de transformación social y emancipador (Apple, 2011; Erazo \& Erazo, 2015; Freire 2003; Ovejero 2004). Pero para ello es necesario avanzar hacia una escuela que primero, ha de transformarse a sí misma a fin de superar su anacronismo histórico, para luego también ser capaz de formar ciudadanos autónomos, críticos y responsables de hacer prevalecer en un futuro una sociedad igualitaria y democrática. Por tanto, una institución capaz de oponerse y cuestionar las formas de socialización, los valores dominantes y el "pensamiento único". Instaurando en consecuencia, a la escuela como una instancia de construcción de la sociedad y el conocimiento, transformándose en un espacio dialógico-comunicativo, que fomente la inclusión social y la cooperación mutua (Aubert, Duques, Fisas \& Valls, 2004; Bourdieu,

Obra originalmente publicada en 1964, se referencia la edición empleada para redacción del presente artículo.

Obra originalmente publicada en 1981, se referencia la edición empleada para redacción del presente artículo 
2010; Flecha \& Puigvert, 2002; Freire, 1997; Gómez, Latorre, Sánchez \& Flecha, 2006; McLaren \& Kincheloe, 2008; Ovejero, 2004; Ovejero, 1990; Pujolàs, 2009, 2012).

La globalización neoliberal no es un fenómeno de características ideológicas neutras y tampoco lo son sus consecuencias en el sistema educativo (Bonal, 2009; Díez, 2010; KurthSchai, 2014). Por ello, es necesaria la utilización de recursos y estrategias pedagógicas que cooperen a la superación de los conflictos (principalmente psicosociales) asociados a la globalización económica que se producen en los escenarios educativos; los que suelen manifestarse en el aula a través de distintas formas de exaltación del individualismo, la relativización de aspectos éticos y la negación de la cooperación social (Ovejero, 2004; Schneider, 2015; Wagner \& Shahjahan, 2015).

En virtud de los antecedentes, las estrategias didácticas vinculadas con el desarrollo de la socialización, así como con la construcción de situaciones de aprendizaje de naturaleza democrática, emergen como alternativas coherentes frente a una globalización económica que exacerba el individualismo y promueve desmesuradamente el sentido competitivo en el campo educativo (Casanova, 2010). Concretamente la "cooperación" parece ser uno de los elementos centrales a desarrollar en un escenario como antes el descrito, más aun si se pretende construir una escuela basada en valores sociales, principios inclusivos e interacciones positivas entre sus miembros (Pujolàs, 2012). De hecho Kropotkin (2013) ${ }^{6}$ ha planteado que la cooperación ha sido clave como factor evolutivo de la especies y en particular de la humana, aspecto que el darwinismo social imperante en el sistema escolar no suele considerar (Ovejero, 2004).

El aprendizaje cooperativo, así como otros tipos de estrategias didácticas que fomentan la participación dialógica y el apoyo mutuo entre pares, asoman hoy como alternativas que pueden hacer frente a la irrupción del mercado y la globalización neoliberal (Nikolakaki, 2012). La utilización de este tipo de herramientas pedagógicas se presenta como un potente dispositivo transformador, ante la concepción negativa actual de valores como la cooperación, la solidaridad y el consenso, que fundamentalmente son apreciados como aspectos característicos de la debilidad humana (Aubert et al., 2004; Fisher, 2013; Nikolakaki, 2012; Ovejero, 1990; Ovejero, 2004). A ello se suman las características positivas del aprendizaje cooperativo y las estrategias dialógicas-comunicativas, en torno a la integración, interacción e inclusión social, aspectos fundamentales para superar barreras exclusoras y los problemas de convivencia entre "diferentes" en las sociedades actuales (Bash, 2014; Flecha \& Puigvert, 2002; Ovejero, 2004).

Como hemos podido apreciar, la escuela se encuentra en una posición privilegiada para hacer frente a las repercusiones sociales negativas de la globalización neoliberal. Sin embargo, para ello es imprescindible realizar cambios profundos en el campo educativo, así como en las representaciones existentes respecto a "educación", "escuela", "estudiantes" y las relaciones que se configuran dentro del aula, para así avanzar hacia una pedagogía transformadora, basada en el dialogo y la cooperación. Además emerge la necesidad de concebir una escuela contextualizada a su periodo histórico y al tipo de sociedades en las que se desarrolla, para con ello lograr atender efectivamente las problemáticas contingentes y significativas que afectan a sus miembros y la comunidad en general.

Obra originalmente publicada en 1902, como respuesta en contrapartida al Darwinismo Social. Se referencia la edición empleada para redacción del presente artículo 


\section{PROPUESTAS CRÍTICAS DESDE LA EDUCACIÓN FÍSICA Y EL DEPORTE}

Si bien es innegable que las prácticas tecnocráticas han tenido y siguen teniendo mucha presencia en el área de la EF y el deporte, no es menos cierto que han existido movimientos alternativos que han estudiado, analizado y propuesto alternativas al respecto (Bain, 1993; Fernández-Balboa, 1993a, 1993b, 1993c; 1995, 1997; Devís \& Peiró, 1992, 2007; López Pastor, González \& Barba, 2005; López Pastor et al., 2006a, 2006b; 2007; Pascual, 1999, 2002, 2004; Rivera \& De La Torre, 2005; Trigueros, De la Torre \& Rivera, 2006; De La Torre, Rivera \& Trigueros, 2007; Rivera, Trigueros, De la Torres \& Moreno, 2010). En esta misma línea, Kirk (2001) ha expresado las posibilidades que ofrece éste campo para el desarrollo de espacios vinculados a la reflexión crítica de la realidad:

Una pedagogía crítica busca capacitar y emancipar al profesorado ayudándole a realizar conexiones entre sus propias experiencias de vida y el más extenso, pero menos visible, proceso social como, por ejemplo, la construcción social del género en el deporte o las relaciones entre deporte, dinero, media y poder. También intenta abordar complejas cuestiones y situarlas en las experiencias diarias del profesorado sobre el deporte y la actividad física (2001: 1001-1002).

Desde la Pedagogía Crítica en EF son diversas las problemáticas que se han trabajado durante las últimas décadas. A continuación daremos cuenta sobre algunos de sus ejes vertebradores, lo que nos permitirá una comprensión mayor de las alternativas existentes.

Una de las preocupaciones más relevantes ha sido la democratización de los espacios y tiempos de la EF escolar (Rivera \& De La Torre, 2005; Rivera et al., 2010; Fraile, 2006; López Pastor, 2002). En esta línea han surgido interesantes proyectos como "Democratizar el aula universitaria: formar docentes, formar personas" (Rivera \& De La Torre, 2005). Las ideas claves del proyecto son la consideración de la "no neutralidad" del proceso formativo y la democratización, entendida esta como:

[...] participación con conocimiento de causa, al tiempo que compromiso con la sociedad en la que se enmarca y con una clara finalidad liberalizadora. Nuestra intención es hacer presente el concepto en nuestras clases como si tratara de un estilo de vida, basado en la participación desde el diálogo y la acción cooperativa y planteado desde dos planos complementarios, el ético y el normativo. Evidentemente, nuestro mayor compromiso es el de enseñar valores desde la vivencia. Pensamos que sólo así nuestros alumnos y alumnas tomarán conciencia de lo verdaderamente importante: ayudar a construir ciudadanos y ciudadanas desde el marco que nos ofrece la Educación Física escolar (2005: 94).

Con esta misma idea de la democratización de los espacios educativos, Fraile (20032004), entiende que la educación se está convirtiendo en el instrumento más importante para desarrollar el pensamiento y aumentar la participación en la construcción social, lo que provoca que no tengamos que centrarnos sólo en el cambio de contenidos a enseñar, sino sobre todo en modificar los procesos pedagógicos, democratizando así la actuación de todos los implicados en la acción pedagógica, adecuándonos a las exigencias actuales de co-construcción social. La democratización educativa en EF pasa por desarrollar, entre los 
estudiantes, un interés personal y crítico por lo social. Los principios en que Fraile (20032004) sustenta su propuesta de democratización educativa son:

(a) mayor preocupación por los problemas morales relacionados con los derechos de los estudiantes; (b) una mayor interacción con el alumnado superando el miedo a perder el control de la clase y las relaciones de poder; (c) una mayor atención a la diversidad de los estudiantes; (d) que el conocimiento del profesor no debe representar el principal saber (e) la negociación debe representar un procedimiento didáctico que ayude al docente y discente a interactuar e intercambiar mensajes, códigos y discursos (2003-2004: 219-220).

La idea es mantener una reflexión crítica constante, que permita de-construir las conceptualizaciones existentes, que están sustentadas y ancladas en el paradigma tecnocrático de la enseñanza y el aprendizaje en EF. Para ello es necesario construir un proceso de dialógico de co-construcción de conocimientos negociados y consensuados surgidos a partir del trabajo colaborativo de todos los implicados en la acción pedagógica y en un proceso autoevaluativo, que permita ser autocrítico con los propios aprendizajes y procesos vividos (Fraile, 2006).

El aprendizaje cooperativo, como metodología para la democratización enunciada otorga interesantes posibilidades (Fraile, 2008):

(a) ayuda a superar las tendencias individualistas y competitivas que caracterizan la actuación de los estudiantes; (b)incentiva la mejora de la comunicación entre el profesorado y los estudiantes; (c) su finalidad es fomentar entre los participantes las habilidades sociales que ayuden a conectar sus intereses con la práctica, incidiendo en su motivación, autonomía y responsabilidad (2008: 3-4).

Una EF que se aleja de una intencionalidad educativa basada en la generación de autonomía, pensamiento crítico y capacidad reflexiva de los estudiantes no es una EF crítica. Estrategias metodológicas y prácticas evaluativas no acordes con estas intencionalidades no son procedimientos críticos en el contexto escolar.

López Pastor $(1999,2014)$ propone lo que él denomina una evaluación formativa. En esta propuesta, anclada en una lógica crítica, la corrección se convierte en un instrumento de aprendizaje y no de castigo; y la comprensión del error es fuente misma de aprendizaje. Además de formativa, la evaluación también debe ser compartida, es decir, debe convertirse en un proceso democrático de diálogo y toma de decisiones colegiadas y compartidas. En términos resumidos, Lopéz Pastor et al. (2006) entienden la evaluación como un espacio de comprensión y mejora, es decir una herramienta al servicio de los procesos educativos del alumnado y de la construcción de procesos democráticos en el aula:

Por evaluación formativa entendemos todo proceso de evaluación cuya finalidad principal es mejorar los procesos de enseñanza-aprendizaje [...] es todo proceso de evaluación que sirve para que el alumnado aprenda más (y/o corrija sus errores) y para que el profesorado aprenda a trabajar mejor (a perfeccionar su práctica docente). Por decirlo de otro modo, la finalidad no es calificar al alumno, sino disponer de información que permita saber cómo ayudar al alumnado a mejorar y aprender más [...] y que sirva 
a su vez para que los profesores aprendamos a hacer nuestro trabajo cada vez mejor. Por evaluación compartida entendemos que la evaluación debe ser más un diálogo y una toma de decisiones mutuas y/o colectivas, que un proceso individual e impuesto. Dentro de estos procesos las autoevaluaciones, las coevaluaciones y las evaluaciones y calificaciones dialogadas son técnicas que juegan un papel fundamental (2006: 37).

Dialogar y consensuar colegiadamente requiere de una verdadera participación del alumnado en la toma de decisiones a seguir, por lo que parte del trabajo crítico en EF está orientado hacia la autonomía y mejora en el aprendizaje, el pensamiento crítico, la responsabilidad y educación democrática, así como la coherencia personal y profesional (López Pastor, 2004).

En estas propuestas orientadas por la Pedagogía Crítica, o por sus principios de acción, se hace necesario e indispensable un análisis contextual que ponga en relación criterios políticos, económicos, socioculturales y educativos característicos de la sociedad donde vivimos y así no caer en contradicciones personales y profesionales, pues educar en democracia es fácil de decir, pero en muchas ocasiones la "realidad práctica" nos puede hacer develar muchas prácticas educativas antidemocráticas (Pascual, 1999). Es por ello que se propone el trabajo pedagógico en EF a partir de la dignidad y responsabilidad con uno mismo y con los estudiantes. La EF, en sus diferentes niveles, ha dado la espalda a algunas temáticas relevantes en la labor educativa. Por ejemplo, el culto al cuerpo y a la esbeltez, baja autoestima, timidez, problemas de comunicación, conductas adictivas, necesario respeto para la convivencia, desarrollo del patriotismo, el nacionalismo, la identidad a través del deporte, etc. (Pascual, 1999).

Es así como el profesor de EF debiera convertirse en un profesional del sistema educativo con amplios conocimientos sobre aquello que va a enseñar, pero ello acompañado de las características de madurez y equilibrio emocional, compromiso con su función, preocupación por la persecución de fines valiosos en sí mismos, capaces de justificar sus acciones desde criterios éticos y que utilice estrategias metodológicas coherentes con esos fines. Para Pascual, ello sería "una persona que está en proceso continuo de cuestionamiento y mejora de sus prácticas (como persona y como profesional), [...] alguien capaz de mejorar a través de la praxis [...] en un proceso de reflexión-acción- reflexión" (1999: 133).

El trabajo pedagógico del profesor de EF debe estar orientado por un profesor investigador de su propia práctica, que reflexione sobre ella. Es por ello que la acción educativa debe estar estrechamente vinculada a la ética profesional, en el sentido del arte de realizar bien el trabajo (Pascual, 2004).

Esta reflexión profesional acerca de las creencias, concepciones y teorías acerca de la EF escolar es la que lleva a algunos autores (Devís, 1995; Fraile, 2001) a proponer una reinterpretación de algunos contenidos propios del área de EF, siempre con una visión crítica de los mismos. Es así, como por ejemplo, se plantea una fuerte crítica al deporte escolar tradicional y se proponen algunos cambios sustanciales al uso que se hace de él en el contexto escolar. Hay un cuestionamiento sobre las creencias que sostienen la importancia de la competición para el desarrollo personal y social, así como sobre los intereses que mantienen y promocionan un deporte escolar con competiciones y campeonatos extraescolares.

Devís (1995) propone que el deporte escolar debe "estar centrado en una educación deportiva dirigida al cambio y la transformación de las experiencias y estructuras del deporte" (p. 462). Ello conduciría a cuestionar, críticamente, el concepto de competición 
deportiva, atendiendo a la discriminación en función del sexo y en función de las diferentes capacidades físicas, el contexto social, político y económico que genera un tipo de deporte determinado. En este cambio no es posible que el profesor de EF actúe en solitario sino que, por el contrario, debiera realizar un trabajo colaborativo con toda la comunidad educativa, entendiéndola en su sentido más amplio (estudiantes, profesores, padres y madres, administración educativa, etc.).

Esto ayudaría a alivianar las deficiencias propias del modelo tradicional del deporte escolar (centrado muchas veces en la competición exacerbada) y a sustentar su práctica en un modelo caracterizado por una orientación verdaderamente educativa (Fraile, 2001). En este contexto del deporte y, más concretamente, de su aplicación en el contexto escolar es interesante la propuesta investigativa de Soler (2009), pues centrándose en el fútbol, como deporte más practicado por los jóvenes, realiza un análisis en función de las prácticas sexistas que en él se generan y las dinámicas de reproducción de estereotipos tradicionales de género. La autora se pregunta por las posibilidades que tiene la asignatura de EF de constituirse en un espacio y tiempo pedagógico que ayude en la transformación de los modelos dicotómicos y sexistas de masculinidad y feminidad.

Los estudios de Soler $(2007,2009)$ dan cuenta de que si bien es posible observar una clara discriminación para la mayor parte de las niñas y para algunos niños, también es posible percatarse de algunas actitudes de resistencia al modelo deportivo tradicional, lógicas emergentes en las sociedades actuales; precisamente en una de sus investigaciones (Soler, 2009) menciona: “[...] se observa cómo las niñas no aceptan sin más su situación, sino que muestran su inconformismo y rebeldía" (p. 40). Es por esto que la autora afirma y enfatiza la importancia de la creación de espacios para la reflexión entre alumnos y profesores, pues ello ayudaría a que el docente pudiera generar una visión crítica de su propio quehacer.

Otro contenido importante cuestionado por varios autores es el de la "salud" (Colquhoun, 1990; Devís, 1992; Sparkes, 1991, 2004), considerando que la forma tradicional de entender el concepto de salud en el área de EF está asentado en una serie de creencias encubiertas que nos hacen creer que la salud es algo individual y personal, responsabilizando a las personas de la buena o mala salud que estas puedan poseer. Los medios de comunicación y las nuevas tecnologías nos llenan de imágenes a partir de las cuales aprendemos que "salud" y "belleza" estética mantienen un relación directa, con las consecuencias que dicha relación puede tener sobre los adolescentes (Toro, 2004). Tirado y Ventura (2009) anuncian el peligro de las ideas subyacentes en estos mensajes, a partir de los cuales se puede construir la idea de que las personas con "cuerpos bellos" son las que consiguen construir cierto éxito social, dando a conocer que muchos de los problemas emocionales en la adolescencia se inician en la falta de coherencia entre la imagen que tienen de ellos mismo y lo que la lógica social nos transmite.

Se propone la realización de una reinterpretación de los significados asociados al cuerpo, el ejercicio, el deporte, el sexo, la misma salud y los mismos estilos de vida propios de la sociedad consumista actual en donde nos desarrollamos (Devís \& Peiró, 1992). La intencionalidad está determinada por entender que los problemas de salud se contextualizan en los espacios y tiempos sociales y ambientales y, por tanto, las estrategias que utilicemos en la EF escolar para el trabajo de la salud deben ir dirigidas a facilitar el cambio social y comunitario (Peiró \& Devís, 2001) a través de una práctica física reflexionada y conceptualizada (Pérez, Delgado \& Rivera, 2009). 
Estudios Pedagógicos XLIII, Nº 3: 103-117, 2017

GLOBALIZACIÓN ECONÓMICA, POSTMODERNIDAD Y SISTEMA EDUCATIVO: CONTRADICCIONES Y

ALTERNATIVAS DESDE UNA EDUCACIÓN FÍSICA CRÍTICA

Tirado y Ventura (2009) proponen el desarrollo de la competencia crítica en el alumnado de EF, a partir de una experiencia en el sistema educativo, dirigida a construir esa visión crítica sobre las prácticas deportivas tradicionales que exacerba, entre otras cosas, el culto al cuerpo como sinónimo de salud.

Para que esta intencionalidad crítica llegue a operacionalizarse y consigamos, así, convertir a la EF en un área de conocimientos verdaderamente implicada en la transformación de las sociedades actuales que tienen el cambio rápido e incierto como una de sus características principales, es necesario realizar un análisis de las preconcepciones ideológicas que sustentan las prácticas más anacrónicas y tradicionales en la EF escolar, tomar conciencia en relación a las prácticas opresivas y represivas y crear estrategias para redefinir y transformar las circunstancias y el contexto (Fernández-Balboa, 1995, 1999).

Esto conllevaría no dejar de lado los aspectos políticos y éticos de la labor pedagógica, atendiendo a que el proceso de enseñanza-aprendizaje en la EF no puede limitarse al qué y cómo enseñar, sino que se deben desarrollar los por qué y para qué de dicho proceso, pues de esa forma se podrán desarrollar las capacidades morales, éticas y políticas de los futuros ciudadanos (Fernández-Balboa, 2004). Esto nos invita a develar el curriculum oculto de la EF, atendiendo a las características de estratificación y jerarquización, elitismo, discriminación, bajísima relevancia del contexto educativo, fragmentación del conocimiento, competitividad e individualismo, lógica lineal, restrictiva y reproductiva, estandarización y no personalización, prácticas supuestamente neutrales y apolíticas, e instancias coercitivas (Fernández Balboa, 1993b). De lo contrario, todas estas características terminan siendo fuertes barreras en la construcción de una EF alternativa a la tradicional, en definitiva más humana y más crítica. Una fuerte barrera con la que nos encontramos los profesionales del área es el supuesto aspecto científico de la enseñanza (Fernández-Balboa, 2004). La comprensión de lo oculto, lo no abordado pedagógicamente de forma explícita por el profesorado de EF, se hace indispensable si queremos construir una labor docente dirigida a desarrollar las capacidades morales, éticas y políticas de los futuros ciudadanos.

Para terminar, nos resulta de suma importancia mostrar algunas líneas de acción concretas, propuestas por Fernández-Balboa (1999) para la transformación de las condiciones que oprimen en el contexto de la EF:

- Traspasar barreras artificiales: los espacios educativos están estructurados dadas las ideologías que subyacen a dicha estructuración, de forma tal que genera la separación de niños y niñas para el desarrollo de las actividades de clase. Un ejemplo recurrente y común de ello es la práctica del fútbol, espacio en que los niños suelen participar y las niñas se excluyen de dicha actividad. Esta es una separación construida socialmente $\mathrm{y}$, por tanto, se le invita a los docentes a que sean capaces de observar y preguntarse críticamente por esta realidad pedagógica de los patios y aulas escolares, así como de otros espacios sociales

- Agresividad y violencia en el deporte: por todos es reconocido que la agresividad y la violencia tienen mayor presencia en los espectáculos deportivos de la que desearíamos. La relación entre estos aspectos y la masculinidad, las diferentes teorías sobre la agresión, la construcción de patrones culturales, etc., podrían ser temas de reflexión y cuestionamiento en las clases de EF

- Aficionados y forofos (tipos, actividades y propósitos): la afición a las actividades deportivas, ya sea como practicante o como espectador es algo extendido entre la pobla- 
ción mundial (Antolín, de la Gándara, García \& Martín, 2009; Prat \& Gómez, 2009), por lo que sin duda es una realidad significativa para gran parte del alumnado de EF. Es por ello que el autor nos propone el análisis de cómo esta afición influye en la vida de las personas en sus diferentes dimensiones: cultural, económica, social, histórica, psicológica, etc.

- El cuerpo humano: entender el cuerpo desde su vertiente biofisiológica y excluir el entendimiento del mismo desde una visión social construida en EF se constituye en una limitación de nuestra área de estudio. ¿Quiénes se benefician o perjudican con la visión reduccionista del cuerpo?, ¿qué valores transmiten los medios de comunicación en relación al cuerpo a través de las imágenes y mensajes corporales?, ¿quién determina lo que es cuerpo saludable? Estas son algunas de las preguntas que nos propone el autor para trabajar con el alumnado de EF.

- Género y sexualidad en el deporte: significados de lo que es ser hombre y mujer, la relación entre la sexualidad y el género, característica físicas, psicológicas y sociales asociadas a las masculinidad y feminidad, las relaciones de poder que se generan a través del género, todo ello en el contexto deportivo, son temáticas y cuestionamientos que debieran ser abordados pedagógicamente desde el área de EF, pues se hace necesario una reconstrucción de las ideas previas relacionadas con estos tópicos.

- Discurso y simbología en el deporte: el sistema de creencias (ideología) que sustenta nuestro accionar es construido a partir del lenguaje y los símbolos. Es por ello que develar el discurso y los símbolos asociados al ámbito deportivo se hace necesario para comprender cuáles son las intencionalidades ligadas a dicho contexto y al uso que los medios de comunicación hacen del mismo.

\section{REFERENCIAS BIBLIOGRÁFICAS}

Abril, A. (2015). Ciudadanía, educación y complejidad: miradas desde la econopedagogía. Diálogo Andino, 47, 95-104.

Antolín, V., de la Gándara, J., García, I., \& Martín, A. (2009). Adicción al deporte: ¿moda postmoderna o problema sociosanitario?. Revista norte de salud mental, 8(34), 15-22.

Apple, M. (2011). Democratic education in neoliberal and neoconservative times. International studies in sociology education, 21(1), 21-31.

Apple, M. (2013a). Can education change society? New York: Routledge.

Apple, M. (2013b). Education and Power. New York: Routledge.

Aubert, A., Duque, E., Fisas, M., \& Valls, R. (2004). Dialogar y transformar: Pedagogía Crítica del Siglo XXI. Barcelona: GRAÓ.

Bain, L. (1993). Ethical issues in teaching. Quest, 45, 69-77.

Bash, L. (2014). Some Issues for Cooperative Learning and Intercultural Education: Reflections on Aspects of the Recent Work of Jagdish Gundara. Intercultural education, 25(3), 179-186.

Bonal, X. (2009). La educación en tiempos de globalización: ¿quién se beneficia?. Educação \& Sociedade, 30, 653-671.

Bourdieu, P. (2007). Razones prácticas. Barcelona: Anagrama.

Bourdieu, P. (2010). Capital cultural, escuela y espacio social. Ciudad de México: Siglo XXI.

Bourdieu, P., \& Passeron, J. (1990). Reproduction in education, society and culture, London: Sage.

Bourdieu, P., \& Passeron, J. (2009). Los herederos. Buenos Aires: Siglo XXI.

Cardona, L. (2014). Sistemas de creencias en transformación de aulas. Modelos pedagógicos para la 
Estudios Pedagógicos XLIII, N 3: 103-117, 2017

GLOBALIZACIÓN ECONÓMICA, POSTMODERNIDAD Y SISTEMA EDUCATIVO: CONTRADICCIONES Y

ALTERNATIVAS DESDE UNA EDUCACIÓN FÍSICA CRÍTICA

transmodernidad. Sophia, 10(1), 145-150.

Caro, J. (2013). La democracia cansada: globalización, postmodernidad y multiculturalismo. Bajo palabra: Revista de filosofía, 8, 237-246.

Casanova, F. (2010). El aprendizaje cooperativo y los valores bioéticos en educación: opción entre globalización o mundialización. Revista Colombiana de Bioética, 5(2), 53-60.

Colquhoun, D. (1990). Images of healthism in health based physical education. En D. Kirk \& R. Tinning (Eds.), Physical education, curriculum and culture: critical issues in the contemporary crisis (pp. 225-251). London: Taylor \& Francis.

De La Torre, E., Rivera, E., \& Trigueros, C. (2007). Creencias y concepciones de la educación física en evolución: el caso de la formación del profesorado de educación física en la educación primaria. Tándem, 23, 50-56.

Devís, J. (1995). Deporte, Educación y Sociedad. Hacia un deporte escolar diferente. Revista de Educación, 306, 455-472.

Devís, J., \& Peiró, C. (1992). Nuevas perspectivas curriculares en educación física: la salud y los juegos modificados. Barcelona: Inde.

Devís, J., \& Peiró, C. (2007). La creación del espacio europeo de educación superior y la formación de los profesionales de la educación física y el deporte en España: iniciativas desde la Universidad de Valencia. L. Martínez \& N. Borés (Eds.), La Formación del profesorado de Educación Física en el marco de la convergencia europea (pp. 85-106). Valladolid: Universidad de Valladolid.

Díez, E. (2010). La globalización neoliberal y sus repercusiones en la educación. Revista electrónica interuniversitaria de formación del profesorado, 13(2), 23-38.

Erazo, V., \& Erazo, L. (2015). Modernidad, desarrollo y educación: de las causas de los civilizatorio hacia la crisis civilizatoria. Tendencias, 16(1), 99-124.

Fernández-Balboa, J. (1993a). Critical pedagogy: Making critical thinking really critical. Analytic Teaching, 13(2), 61-72.

Fernández-Balboa, J. (1993b). Aspectos crítico y cívico del rol de los/as profesionales de la Educación Física y el deporte: Conexiones con la política, la economía y el medio ambiente. Apunts, 34, 74-82.

Fernández-Balboa, J. (1993c). Sociocultural characteristics of de hidden curriculum in physical education. Quest, 45(2), 230-254.

Fernández-Balboa, J. (1995). Reclaiming physical education in higher education through critical pedagogy. Quest, 47(1), 91-114.

Fernández-Balboa, J. (1997). Physic Sociocultural characteristics of the hidden curriculum in physical educational education teacher preparation in the postmodern era: Toward a critical pedagogy. En J. Fernández-Balboa (Ed.), Critical postmodernism, human movement, physical education and sport (pp.121-138). Albany: SUNY.

Fernández-Balboa, J. (1999). Pedagogía Crítica y Educación Física en la Escuela Secundaria. Revista Conceptos de Educación, 6, 15-32.

Fernández-Balboa, J. (2004). Recuperando el valor ético-político de la Pedagogía: las diferencias entre la Pedagogía y la Didáctica. En A. Fraile (Ed.), Didáctica de la Educación Física. Una perspectiva crítica y transversal (pp. 315-330). Madrid: Editorial Biblioteca Nueva.

Feyerabend, P. (1986). Tratado contra el método. Madrid: Editorial Tecnos.

Feyerabend, P. (1990). Diálogo sobre el método. Madrid: Cátedra.

Fisher, R. (2013). Dialogo creativo: hablar para pensar en el aula. Madrid: Morata.

Flecha, R. (2001). Racismo moderno y postmoderno en europa: enfoque dialógico y pedagogías antirracistas. Reis: Revista española de investigaciones sociológicas, 94, 79-103.

Flecha, R., \& Puigvert, L. (2002). Multiculturalismo y Educación. En T. Lleixà (Ed.), Multiculturalismo y Educación Física (pp. 9-43). Barcelona: Paidotribo.

Foucault, M. (2007). Un dialogo entre el poder y otras conversaciones. Madrid: Alianza.

Fraile, A. (2001). Actividad Física Jugada. Una propuesta educativa para el deporte escolar. 
Alicante: Marfil.

Fraile, A. (2003-2004). Un cambio democrático en las aulas universitarias. Una experiencia en la formación del profesorado de Educación física. Revista Contextos Educativos, 6-7, 213-234.

Fraile, A. (2004). Didáctica de la Educación Física. Una perspectiva crítica y transversal. Madrid: Biblioteca Nueva.

Fraile, A. (2006). Cambios en el aula universitaria ante los nuevos retos europeos. Tándem, 20, 57-72.

Fraile, A. (2008). El aprendizaje cooperativo como metodología para el desarrollo de los ects: una experiencia de formación del profesorado de Educación Física. Fuentes, 8, 22-35.

Freire, P. (1997). Política y Educación. Madrid: Siglo XXI.

Freire, P. (2003). El grito manso. Buenos Aires: Siglo XXI.

Freire, P. (2012). Pedagogía del oprimido. Ciudad de México: Siglo XXI.

Gómez, J., Latorre, A., Sánchez, M., \& Flecha, R. (2006). Metodología comunicativa crítica. Barcelona: El Roure.

Hargreaves, A. (2005). Profesorado, cultura y postmodernidad. Madrid: Morata.

Khun, T. (2010). La estructura de las revoluciones científicas. Ciudad de México: Fondo de Cultura Económica.

Kirk, D. (2001). Fundamentos para una pedagogía crítica en la formación del profesorado de Educación Física. En J. Devís (Ed.), La Educación Física, el deporte y la salud en el siglo XXI. España: Marfil.

Kropotkin, P. (2013). El apoyo mutuo: un factor en la evolución. Madrid: Dharama.

Kurth-Schai, R. (2014). Fidelity in Public Education Policy: Reclaiming the Deweyan Dream. Educational Studies, 50(5), 420-446.

Londoño, A. (2008). Globalización, cultura y posmodernidad: la emergencia de un nuevo sujeto. Criterios, 1(2), 39-54.

López Pastor, V. (1999). Prácticas de evaluación en Educación Física: estudio de casos en primaria, secundaria y formación del profesorado. Valladolid: Universidad de Valladolid.

López Pastor, V. (2002). Algunas reflexiones sobre Educación Física y pedagogía crítica. Retos, 2, 30-35.

López Pastor, V. (2004). La participación del alumnado en los procesos evaluativos: la autoevaluación y la evaluación compartida en Educación Física. En A. Fraile (Ed.), Didáctica de la Educación Física. Una perspectiva crítica y transversal (pp. 265-291). Madrid: Editorial Biblioteca Nueva.

López Pastor, V. (2014). Nuestra propuesta de evaluación en Educación Física: por una evaluación formativa y compartida. En V. López Pastor (Ed.), La evaluación en Educación Física. Revisión de los modelos tradicionales y planteamiento de una alternativa: La evaluación formativa y compartida (pp. 75-100). Buenos Aires: Miño y Dávila.

López Pastor, V., Barba, J., Monjas., R., Manrique, J., Heras, B., González, M., \& Gómez, J. (2007). Trece años de evaluación compartida en Educación Física. Revista Internacional de Medicina y Ciencias de la Actividad Física y del Deporte, 7(26), 69-86.

López Pastor, V., González, M., \& Barba, J. (2005). La participación del alumnado en la evaluación: la autoevaluación, la coevaluación y la evaluación compartida. Tandem, 17, 21-37.

López Pastor, V., Monjas, R., Gómez, J., López, E., Martín, J., González, J., Barba, J., Aguilar, R., González, M., Heras, C., Martín, M., Manrique, J., Subtil, P., \& Marugan, L. (2006a). La evaluación en Educación Física: revisión de modelos tradicionales y planteamiento de una alternativa. Evaluación formativa y compartida. Retos, 10, 31-41.

López Pastor, V., Monjas, R., Gómez, J., López, E., Martín, J., González, J., Barba, J., Aguilar, R., González, M., Heras, C., Martín, M., Manrique, J., Subtil, P., \& Marugan, L. (2006b). La evaluación formativa y compartida en Educación Física. De la crítica al modelo tradicional a la generación de un sistema alternativo. Revisión de 12 años de experiencia. Lecturas Educación Física y Deportes, 10(94),

Lyotard, J. (2000). La condición postmoderna. Barcelona: Gedisa. 
Estudios Pedagógicos XLIII, N 3: 103-117, 2017

GLOBALIZACIÓN ECONÓMICA, POSTMODERNIDAD Y SISTEMA EDUCATIVO: CONTRADICCIONES Y

ALTERNATIVAS DESDE UNA EDUCACIÓN FÍSICA CRÍTICA

Martín, M. (2014). Formación del profesorado en la era postmoderna: una perspectiva narrativa. Revista de Educación, 5(7), 75-92.

McLaren, P., \& Kincheloe, J. (2008). Pedagogía Crítica: De qué hablamos, dónde estamos. Barcelona: GRAÓ.

Munck, R. (2010). La teoría crítica del desarrollo: resultados y prospectiva. Migración y desarrollo, 18(4), 35-57.

Nikolakaki, M. (2012). Building a Society of Solidarity through Critical Pedagogy: Group Teaching as a Social and Democratic Too. Journal for Critical Education Policy Studies, 10(2), 392-417.

Ovejero, A. (1990). El aprendizaje cooperativo: Una alternativa eficaz a la enseñanza tradicional. Barcelona: Promociones y publicaciones universitarias.

Ovejero, A. (2002). Escuela y postmodernidad: reflexiones sobre los actuales problemas. Revista de psicología social aplicada, 12(2), 67-86.

Ovejero, A. (2004). Globalización, sociedad y escuela: cómo hacer frente a los principales problemas actuales de la psicología social crítica. Valladolid: Universidad de Valladolid.

Ovejero, A. (2008). Desigualdad, subdesarrollo y pobreza en la actual globalización ultraliberal. Eikasia, 3(18), 107-177.

Pascual, C. (1999). La formación inicial del profesorado de Educación Física: en busca del significado profesional perdido. Revista Conceptos de Educación, 6, 123-135.

Pascual, C. (2002). La pedagogía crítica en la formación del profesorado de Educación Física. Sobre todo una pedagogía ética. Revista Interuniversitaria de Formación del Profesorado, 43, 123 135.

Pascual, C. (2004). Formación del profesorado: reflexión-acción y la ética del trabajo bien hecho. Tándem, 15, 18-25.

Peiró, C., \& Devís, J. (2001). Fundamentos para la promoción de la actividad física relacionada con la salud. En J. Devís (Ed.), La Educación Física, el deporte y la salud en el siglo XXI (pp. 295322). Alicante: Marfil.

Pereda, C., Actis, W., \& Prada, M. (2000), La inmigración extranjera en España. Barcelona: Caixa.

Pérez, I., Delgado, M., \& Rivera E. (2009). Efectos de un juego de rol sobre los procedimientos de práctica de actividad física relacionada con la salud en secundaria. Profesorado, Revista de currículum y formación del profesorado, 13(3), 317-328.

Prat, M., \& Gómez, I. (2009). Educación Física y entorno social: influencias y repercusiones para propuestas curriculares y educativas. Cultura y Educación, 21(1), 19-30.

Prior, Á. (2002). Axiología de la modernidad. Valencia: Ediciones Cátedra.

Pujolàs, P. (2009). Nueve ideas clave: el aprendizaje cooperativo. Barcelona: GRAÓ.

Pujolàs, P. (2012). Aulas inclusivas y aprendizaje cooperativo. Educatio Siglo XXI, 30(1), 89-112.

Rengifo-Millán, M. (2015). La globalización de la sociedad del conocimiento y la trasformación universitaria. Revista Latinoamericana de Ciencias Sociales, Niñez y Juventud, 13(2), 809-822.

Rivera, E., \& de La Torre, E. (2005). Democratizar el aula universitaria: una propuesta alternativa de formación inicial universitaria desde la participación del alumnado. Investigación en la Escuela, 57, 85-95.

Rivera, E., Trigueros, C., de la Torre., \& Moreno, A. (2010). Formar docentes, formar personas: una experiencia transdisciplinar para democratizar el aula. En L. Álvarez., Rickeman, R., \& Vallès, J (Eds.), Actas del II Congrés Internacional de Didáctiques (pp. 1-8). Girona: Universitat de Girona.

Rivero, R. (2013). Educación y pedagogía en el marco del neoliberalismo y la globalización. Perfiles educativos, 35(142), 149-166.

Ruiz de Samaniego, A. (2004). La inflexión posmoderna: los márgenes de la modernidad. Madrid: Akal.

Schneider, S. (2015). Teaching Neoliberalism, in the Context of Corporate Reform, in the Undergraduate Social Foundations Classroom. Educational Studies: Journal of the American 
Educational Studies Association, 51(4), 284-299.

Sloterdijk, P. (2014). En el mundo interior del capital: para una teoría filosófica de la globalización. Madrid: Siruela.

Soler, S. (2007). Actitudes y relaciones de las niñas y niños ante contenidos de la Educación Física de primaria estereotipado por el género: el caso del fútbol. En B. Vásquez (Ed.), Las mujeres jóvenes y las actividades físico-deportivas (pp. 120-150). Madrid: CSD.

Soler, S. (2009). Los procesos de reproducción, resistencia y cambio de las relaciones tradicionales de género en la Educación Física. Cultura y Educación, 21(1), 31-42.

Sparkes, A. (1991). Alternative visions of health-related fitness: an exploration of problem-setting and its consequences. En N. Amstrong \& A. Sparkes (Eds.), Issues in physical education. London: Casell.

Sparkes, A. (2004). From performance to impaiment: a parchwork of embodied memories. En J. Evans., B. Davies., \& J. Wrigth (Eds.), Body knowledge and control: studies in the sociology of physical education and health (pp. 157-172). London: Routledge.

Teodoro, A. (2014). Critique et utopie, ou une pédagogie de la possibilité dans la construction de politiques d'éducation démocratique. Revista Lusófona de Educação, 26, 125-139.

Tirado, M., \& Ventura, C. (2009). Propuestas para el desarrollo de la competencias crítica en el alumnado de Educación Física. Cultura y Educación, 21, 55-66

Toro, J. (2004). Riesgos y causas de la anorexia nerviosa. España: Ariel.

Trigueros, C., de la Torre, E., \& Rivera, E. (2006). Aprendizaje colaborativo en la formación de Maestros. Una experiencia práctica. Tandem, 20, 45-56.

Wagner, A., \& Shahjahan, R. (2015). Centering Embodied Learning in Anti-Oppressive Pedagogy. Teaching in Higher Education, 20(3), 244-254.

Zeraoui, Z. (2000). Modernidad y Posmodernidad: la crisis de los paradigmas y los valores. Ciudad de México: Limusa. 
